\title{
The association between accelerometer-measured patterns of sedentary time and health risk in children and youth: results from the Canadian Health Measures Survey
}

Rachel C Colley ${ }^{1,2^{*}}$, Didier Garriguet ${ }^{1}$, lan Janssen ${ }^{3,4}$, Suzy L Wong ${ }^{1}$, Travis J Saunders ${ }^{2}$, Valerie Carson ${ }^{3}$ and Mark S Tremblay ${ }^{2}$

\begin{abstract}
Background: Self-reported screen time is associated with elevated health risk in children and youth; however, research examining the relationship between accelerometer-measured sedentary time and health risk has reported mixed findings. The purpose of this study was to examine the association between accelerometer-measured patterns of sedentary time and health risk in children and youth.

Methods: The results are based on 1,608 children and youth aged 6 to 19 years from the Canadian Health Measures Survey (2007-2009). Sedentary time was measured using the Actical accelerometer. Breaks in sedentary time and prolonged bouts of sedentary time lasting 20 to 120 minutes were derived for all days, weekend days and during the after-school period (i.e., after 3 pm on weekdays). Regression analyses were used to examine the association between patterns of sedentary time and body mass index (BMI), waist circumference, blood pressure and non-HDL cholesterol.

Results: Boys accumulated more sedentary time on weekdays after $3 \mathrm{pm}$ and had a higher number of breaks in sedentary time compared to girls. Overweight/obese boys (aged 6-19 years) accumulated more sedentary time after $3 \mathrm{pm}$ on weekdays (282 vs. $259 \mathrm{~min}, \mathrm{p}<.05$ ) and as prolonged bouts lasting at least 80 minutes (171 vs. $133 \mathrm{~min}$, $\mathrm{p}<.05)$ compared to boys who were neither overweight nor obese. Prolonged bouts of sedentary time lasting at least 80 minutes accumulated after 3 pm on weekdays were positively associated with BMl and waist circumference in boys aged 11-14 years $(p<.006)$. Each additional 60 min of sedentary time after 3 pm on weekdays was associated with a $1.4 \mathrm{~kg} \cdot \mathrm{m}^{-2}$ higher BMl and a $3.4 \mathrm{~cm}$ higher waist circumference in 11-14 year old boys. No sedentary pattern variables differed between girls who were not overweight or obese and those who were overweight/obese and none of the sedentary pattern variables were associated with any health markers in girls.
\end{abstract}

Conclusions: The findings confirm results of other studies that reported accelerometer-measured sedentary time was not associated with health risk in children and youth. Even when the pattern and timing of sedentary time was examined relative to health markers, few associations emerged and were limited to boys aged 11-14 years.

Keywords: Behaviour, Breaks, Bouts, Physical activity, Pediatric

\footnotetext{
* Correspondence: rcolley@cheo.on.ca

${ }^{1}$ Health Analysis Division, Statistics Canada, Ottawa, ON, Canada

${ }^{2}$ Children's Hospital of Eastern Ontario Research Institute, Ottawa, ON,

Canada

Full list of author information is available at the end of the article
} 


\section{Background}

The association between sedentary behaviour and health risk in children appears to be influenced by how the sedentary behaviour is measured, defined and categorized. Several studies have reported significant associations between self-reported screen time and increased risk of obesity and cardio-metabolic disease risk in children [1-4]. However, screen time provides a limited perspective on total sedentary time because it is only a sub-component of a behaviour that is defined as encompassing "any waking behaviour characterized by an energy expenditure $\leq 1.5$ METs while in a sitting or reclining position" [5]. Further, self-reported data relating to lifestyle habits may be limited by bias and recall difficulties [6,7].

Accelerometers are now commonly used to objectively measure total sedentary time, and have the capacity to also derive the pattern and timing in which it is accumulated. In contrast to self-report, associations between accelerometer-measured sedentary time and health risk in children have been mixed with some studies reporting significant associations [8-10] and others not [1,11-16]. Studies that collected both questionnaire and accelerometer data on sedentary behaviour/time found an association between self- or parent-reported screen time and health risk but no association between accelerometermeasured sedentary time and health risk $[1,14,15]$. The only studies reporting significant associations between accelerometer-measured sedentary time and health risk did not adjust for moderate-to-vigorous physical activity (MVPA) [8-10] or reported that the significant associations existed in unadjusted models but were attenuated when MVPA was controlled for $[13,16]$. Some engagement in sedentary behaviour is inevitable in the day (e.g., eating, relaxing, homework, school etc.); however, it is presently unknown how much sedentary time is too much. Inevitably, there is variation between people in the length of time they engage in sedentary behaviour bouts (i.e., prolonged periods of sedentary time) and how often these bouts are interrupted by activity. Research in adults suggests that the pattern of accumulation of sedentary time is important to consider in relation to health risk.

The inconsistent findings between accelerometermeasured sedentary time and health risk among children and youth have led to the examination of more sophisticated sedentary time variables. For example, it has been proposed that the pattern in which sedentary time is accumulated may provide insight beyond what has been observed to-date using the total volume of sedentary time [17]. Others have attempted this approach in adults and children; however, the sedentary pattern variables have been limited to breaks or interruptions in sedentary time [17] and engagement in prolonged bouts of sedentary time lasting up to 30 minutes [1]. Further, studies examining how the pattern of sedentary time relates to health risk in children have not considered the importance of periods of discretionary free time separate from the whole day in children [18-20]. The present study sought to build upon this work by extending the length of the prolonged bouts up to 2 hours and by examining these variables during periods when children and youth typically have free time. In other words, this study sought to identify novel sedentary pattern variables that were more representative of how children and youth typically engage in sedentary behaviour.

The purpose of this study was to examine the association between accelerometer-measured patterns of sedentary time and health risk in children and youth. Specifically, this study examines whether breaks in sedentary time and sedentary time accumulated as prolonged bouts during periods of discretionary free time in children (i.e., after-school and weekends) have stronger associations with health risk in children when compared to average daily sedentary time across the week. We hypothesize that sedentary time accumulated during periods of discretionary free time will better discriminate between children engaging in healthy and unhealthy levels of sedentary behaviour when compared to simply examining overall sedentary time.

\section{Methods}

\section{Data source}

The Canadian Health Measures Survey (CHMS) collected data from a nationally representative sample of the population aged 6 to 79 years living in private households at the time of the survey. Data were collected at 15 sites across Canada from March 2007 through February 2009. Ethics approval was obtained from Health Canada's Research Ethics Board [21]. For children aged 6-13 years, written informed consent was obtained from a parent or legal guardian, in addition to written informed assent from the child; youth aged $\geq 14$ years provided independent consent. Of the households selected, $69.6 \%$ agreed to participate. Of that group, $88.5 \%$ of the selected 6-19 year olds completed a questionnaire and $86.9 \%$ of this group participated in the mobile examination centre component. Of the children and youth who agreed to wear the accelerometer and returned the device, $87.4 \%$ had at least one valid day of data, and $76.3 \%$ had at least four valid days. These multiple stages of response can be multiplied together $(69.6 \% \times 88.5 \% \times$ $86.9 \% \times 76.3 \%)$ to provide an overall response rate of $40.8 \%$. Adjustments were made at each stage to manage any potential non-response bias. The data were then weighted to be representative of the Canadian population. More extensive details of the CHMS [22] and direct measurement of physical activity in the CHMS $[23,24]$ are available elsewhere. 


\section{Study procedures}

Upon completion of the mobile examination centre visit, ambulatory respondents were asked to wear an Actical accelerometer (Phillips - Respironics, Oregon, USA) over their right hip on an elasticized belt during their waking hours for seven consecutive days, except when the device could get wet. The Actical measures and records time-stamped acceleration in all directions, providing an index of physical activity intensity. The Actical has been validated to measure physical activity in children [25] and cut-points for sedentary intensity have been proposed for children [26]. The accelerometers were initialized to collect data in 60 -sec epochs.

\section{Accelerometer data reduction}

Participants aged 6 to 19 years with four or more valid days [24], one of which was a weekend day, were included in this analysis (Table 1). A valid day was defined as having 10 or more hours of wear time [24]. Wear time was determined by subtracting non-wear time from 24 hours. Non-wear time was defined as at least 60 consecutive minutes of zero counts, with allowance for two minutes of counts between zero and 100 [24]. For each minute, the level of movement intensity was based on cut-points corresponding to intensity level: sedentary $=<$ 100 counts per minute $(\mathrm{cpm})$ [26]; MVPA $=\geq 1,500 \mathrm{cpm}$ [25]. Minutes of MVPA and sedentary time were summed for each day for each participant.

\section{Sedentary time variables}

Sedentary time was calculated for all days, weekdays and weekend days. The total number of breaks in sedentary time was summed for each valid day and then averaged across the week, weekdays and weekend days. A break was considered as an interruption in sedentary time (lasting a minimum of one minute) in which there was a transition in accelerometer count from $<100 \mathrm{cpm}$ to $\geq$ $100 \mathrm{cpm}$.

To be defined as a prolonged sedentary bout, there had to be $\geq 80 \%$ of minutes below the $100 \mathrm{cpm}$ cut-point (e.g., 16 out of 20 minutes or 32 out of 40 minutes) [1]. The bout stopped when $<80 \%$ was below the $100 \mathrm{cpm}$ cut-point or when there were $\geq 3$ consecutive minutes $\geq 100 \mathrm{cpm}$ or any observations $\geq 1500 \mathrm{cpm}$ (cut-point for moderate intensity). Sedentary bouts lasting at least 20 , $40,60,80,100,120$ minutes were derived using this approach. Multiple lengths of sedentary bouts were derived to reflect a range of different sedentary behaviours such as watching a television show (30 minutes), watching a movie (1.5-2 hours), or playing video games (anywhere between 20 minutes and 2 hours). The choice of $80 \%$ as the criteria for sedentary minutes within a bout was purposeful to mimic real-world situations where largely sedentary pursuits (e.g., watching TV, doing homework) are often occasionally interrupted with light activity (e.g., to go to washroom, answer the phone, get a snack etc.).

\section{Body mass index and waist circumference}

Height was measured to the nearest $0.1 \mathrm{~cm}$ using a ProScale M150 digital stadiometer (Accurate Technology Inc., Fletcher, USA) and weight was measured to the nearest $0.1 \mathrm{~kg}$ with a Mettler Toledo VLC with Panther Plus terminal scale (Mettler Toledo Canada, Mississauga, Canada). BMI was calculated as weight $(\mathrm{kg})$ divided by height squared $\left(\mathrm{m}^{2}\right)$. Children were categorized as not overweight/obese (which includes underweight and healthy weight) or overweight/obese according to ageand sex-specific cut-points [27]. Waist circumference was measured with a stretch-resistant anthropometric tape at the end of a normal expiration to the nearest $0.1 \mathrm{~cm}$ at the mid-point between the last rib and the top of the iliac crest [28].

\section{Blood pressure}

Systolic and diastolic blood pressure were measured with the BpTRU ${ }^{\mathrm{TM}}$ BP-300 device (BpTRU Medical Devices Ltd., Coquitlam, British Columbia); an automated and validated $[29,30]$ electronic monitor that uses an upper arm cuff. Six measurements were taken at 1-min intervals with the last 5 measurements used to calculate

Table 1 Descriptive characteristics of the sample (mean \pm standard deviation)

\begin{tabular}{|c|c|c|c|c|c|c|}
\hline & \multicolumn{3}{|c|}{ Boys } & \multicolumn{3}{|c|}{ Girls } \\
\hline & 6 to 10 years & 11 to 14 years & 15 to 19 years & 6 to 10 years & 11 to 14 years & 15 to 19 years \\
\hline Total sample (n) & 369 & 256 & 184 & 340 & 248 & 211 \\
\hline Age (years) & $8.2 \pm 1.4$ & $12.5 \pm 1.0$ & $17.0 \pm 1.5$ & $8.1 \pm 1.3$ & $12.3 \pm 1.1$ & $16.9 \pm 0.1$ \\
\hline Height (cm) & $133.9 \pm 10.4$ & $158.9 \pm 11.1$ & $175.6 \pm 7.6$ & $131.6 \pm 10.3$ & $156.9 \pm 7.8$ & $166.2 \pm 6.7$ \\
\hline Weight (kg) & $32.5 \pm 9.4$ & $52.1 \pm 14.7$ & $72.4 \pm 18.1$ & $29.9 \pm 8.9$ & $50.6 \pm 11.6$ & $62.5 \pm 13.8$ \\
\hline BMI $\left(\mathrm{kg} / \mathrm{m}^{2}\right)$ & $17.8 \pm 3.1$ & $20.3 \pm 3.9$ & $23.4 \pm 5.0$ & $17.0 \pm 3.1$ & $20.4 \pm 3.8$ & $22.6 \pm 4.4$ \\
\hline Waist circumference (cm) & $61.1 \pm 9.8$ & $70.6 \pm 10.9$ & $80.1 \pm 12.9$ & $57.9 \pm 8.5$ & $70.1 \pm 10.0$ & $75.4 \pm 10.9$ \\
\hline MVPA (average min. $d^{-1}$ ) & $69.4 \pm 29.1$ & $59.5 \pm 29.4$ & $53.1 \pm 25.9$ & $58.1 \pm 22.6$ & $47.2 \pm 24.6$ & $39.1 \pm 23.0$ \\
\hline
\end{tabular}


average blood pressure and heart rate [29]. The device automatically inflates and deflates the cuff and uses an oscillometric technique to calculate systolic and diastolic blood pressure.

\section{Non-HDL-Cholesterol}

Non-HDL-cholesterol was calculated by subtracting HDL cholesterol, measured using a non-HDL precipitation method on the Vitros 5,1FS (Ortho Clinical Diagnostics), from total cholesterol [31]. Non-HDL cholesterol consists of very low density, low density, and intermediate density lipoprotein cholesterol and therefore reflects the cholesterol content of all apo B containing lipoproteins. NonHDL cholesterol was chosen as the lipid marker because it is an important indicator of cardiovascular and diabetes risk among children and adolescents and is not reliant upon a fasted blood sample [32]. Blood samples were taken by a certified phlebotomist and were analyzed at the Health Canada Laboratory (Bureau of Nutritional Sciences, Nutrition Research Division). Other blood markers are available in the CHMS; however, the fasting requirement for some of these measures resulted in a marked reduction in the sample size when they are included. To ensure we had appropriate power for the primary purpose of this analysis, we included non-HDL-cholesterol as the sole blood marker.

\section{Statistical analysis}

Differences between sex, age groups and BMI status were assessed using t-tests. Statistical significance was set at a $p$ value of 0.05 . It is important to note that the values presented from this analysis in Figures 1 and 2 represent the mean across the week for sedentary time accumulated in prolonged bouts. In other words, there are zeros included in the averaging (because not all individuals had bouts of each length on all days) which in the case of 120 minutes bouts, brings the mean time accumulated below 120 minutes.

Associations between sedentary time variables and BMI and waist circumference were assessed using regression analyses. BMI and waist circumference vary by sex and change with normal growth and maturation $[27,33]$. Age and sex were both significantly correlated with average daily minutes of MVPA and age was significantly correlated with average daily minutes of sedentary time. Therefore, all regression analyses were completed separately by sex and the following age categories: $6-10$, 11-14 and 15 to 19 years. The choice of age categories was based on the sampling design of the CHMS. Linear regression models were run separately for each sex and age grouping and were adjusted for age, average daily minutes of MVPA on valid days, and accelerometer wear time. The wear time adjustment was specific to the time period being examined. For example, the models for weekdays after $3 \mathrm{pm}$ where adjusted for wear time on weekdays after $3 \mathrm{pm}$. Eight separate regression models (sedentary time, breaks in sedentary time, prolonged bouts lasting at least 20,40,60,80, 100, 120 minutes) were run for each time period: overall, weekdays after $3 \mathrm{pm}$ and weekends. The $p$-value to reach statistical significance in the linear regression analyses was adjusted for the number of models run. In other words, to reach statistical significance, the regression $p$ values had to be less than .006 (i.e., $0.05 / 8=0.006$ ).

All statistical analyses were performed using SAS v9.1 (SAS Institute, Cary, NC) and were based on weighted data (to be representative of the Canadian population and to account for non-response bias) for respondents with at least four valid days. To account for survey design effects of the CHMS, standard errors, coefficients of

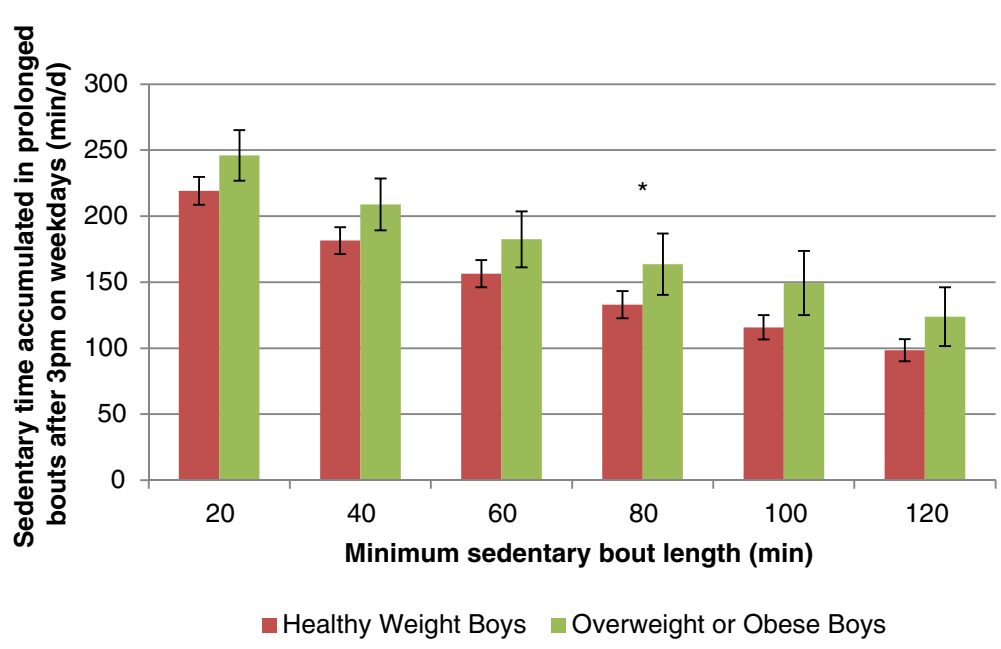

Figure 1 Sedentary time accumulated in prolonged bouts after 3 pm on weekdays in boys. ${ }^{*}$ significant difference between healthy weight and overweight or obese. 


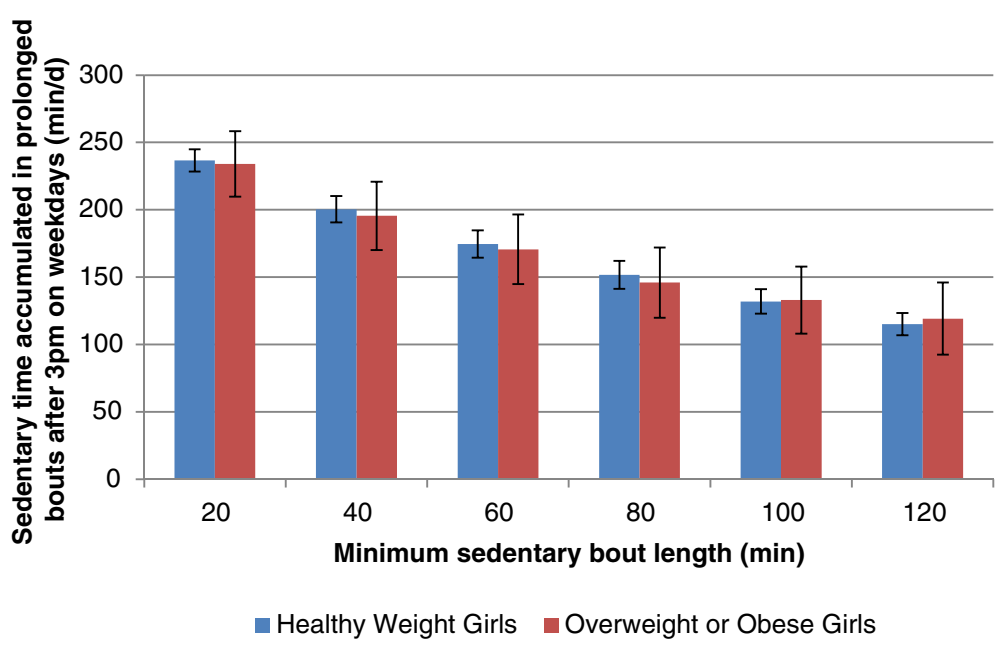

Figure 2 Sedentary time accumulated in prolonged bouts after $3 \mathrm{pm}$ on weekdays in girls.

variation, and 95\% confidence intervals were estimated using the bootstrap technique [34-36]. Overweight and obese were collapsed into one category because we lacked statistical power to compare not overweight/ obese (includes healthy weight and underweight), overweight and obese as 3 separate categories.

\section{Results}

Descriptive characteristics of the sample are provided in Table 1. The analysis is based on 1,608 children and youth between the ages of 6 and 19 years. The sex split was even between boys $(n=809,50.3 \%)$ and girls $(\mathrm{n}=799)$.

\section{Sex and Age differences}

Sex and age differences are presented in Table 2. On average, boys accumulated 508 minutes per day of sedentary time while girls accumulated 524 minutes per day. Boys accumulated more sedentary time on weekdays after $3 \mathrm{pm}$ compared to girls, while boys had a lower number of breaks in sedentary time per day compared to girls. Sedentary time was higher in 11-14 year olds and 15-19 year olds compared to children aged 610 years. Girls aged $15-19$ years accumulated more sedentary time overall and after school compared to boys of the same age.

\section{Body mass index differences}

Differences by BMI status are represented graphically in Figure 1 for boys and in Figure 2 for girls. Overweight and obese boys accumulated more sedentary time after $3 \mathrm{pm}$ on weekdays when compared to boys who are not overweight/obese (Table 2). Overweight and obese boys accumulated more sedentary time after $3 \mathrm{pm}$ on weekdays as prolonged bouts lasting at least 80 minutes when compared to boys who are not overweight/obese (171 vs. $133 \mathrm{~min} \cdot \mathrm{d}^{-1}$ ) (Figure 1). No sedentary time variables differed between girls who are overweight/obese and those who are not overweight or obese (Table 2; Figure 2).

\section{Regression analysis results}

Prolonged bouts of sedentary time lasting at least 40 minutes, after $3 \mathrm{pm}$ on weekdays, were positively associated with waist circumference $(\beta=2.23, p<.006)$ while prolonged bouts of sedentary time lasting at least 80 minutes was positively associated with both $B M I(B=0.72$, $p<.006)$ and waist circumference $(B=1.76, p<.006)$ in boys aged 11-14 years. Each additional 60 minutes of sedentary time accumulated during the after school period was associated with a $1.4 \mathrm{~kg} \cdot \mathrm{m}^{-2}$ higher BMI and a $3.4 \mathrm{~cm}$ higher waist circumference in 11-14 year old boys. Number of breaks in sedentary time, after 3pm on weekdays, was negatively associated with waist circumference $(ß=-$ $4.04, p<.006)$ in boys aged $11-14$ years. No sedentary time variables were significantly associated with BMI or waist circumference in girls of any age or in boys aged 610 or 15-19 years. No sedentary time variables were associated with blood pressure or non-HDL cholesterol in boys or girls. The full results from the regression analyses are available as Additional file 1: Tables S1, Additional file 2: Tables S2, Additional file 3: Tables S3, Additional file 4: Tables S4, Additional file 5: Tables S5.

\section{Discussion}

The objective of this study was to examine the association between accelerometer-measured sedentary time and health risk in children. Our analysis supports previous studies that found few or no significant associations between accelerometer-measured sedentary time and health risk in children [1,11-16]. This study is novel because it 
Table 2 Descriptive sedentary time results (mean \pm standard deviation), by age, sex and obesity status

\begin{tabular}{|c|c|c|c|c|}
\hline & $\begin{array}{l}\text { Sedentary time } \\
(\min / \mathrm{d})\end{array}$ & $\begin{array}{l}\text { Sedentary time on weekdays after } \\
3 \mathrm{pm}(\mathrm{min} / \mathrm{d})\end{array}$ & $\begin{array}{l}\text { Sedentary time on } \\
\text { weekends }(\min / \mathrm{d})\end{array}$ & $\begin{array}{c}\text { Breaks in sedentary time per day } \\
\text { (number/d) }\end{array}$ \\
\hline Boys & $507.5 \pm 90.8$ & $265.5 \pm 65.8^{*}$ & $490.9 \pm 114.5$ & $81.2 \pm 11.6^{*}$ \\
\hline \multicolumn{5}{|l|}{ Age } \\
\hline 6 to 10 years & $445.5 \pm 79.5$ & $212.8 \pm 52.0$ & $440.5 \pm 101.1$ & $84.4 \pm 10.3^{*}$ \\
\hline 11 to 14 years & $524.1 \pm 76.9^{* *}$ & $273.9 \pm 58.0^{* *}$ & $503.2 \pm 103.3^{* *}$ & $79.2 \pm 11.2^{* * * *}$ \\
\hline 15 to 19 years & $553.9 \pm 77.0^{* * * *}$ & $310.7 \pm 44.7^{* * * *}$ & $533.8 \pm 126.4^{* *}$ & $80.1 \pm 13.6^{* *}$ \\
\hline \multicolumn{5}{|l|}{$B M I$} \\
\hline $\begin{array}{l}\text { Not overweight or } \\
\text { obese }\end{array}$ & $499.9 \pm 88.4$ & $259.4 \pm 63.7$ & $483.7 \pm 115.0$ & $81.2 \pm 11.3$ \\
\hline $\begin{array}{l}\text { Overweight or } \\
\text { obese }\end{array}$ & $527.8 \pm 94.6$ & $281.7 \pm 69.2^{* * *}$ & $509.7 \pm 220.0$ & $81.1 \pm 12.6$ \\
\hline Girls & $523.8 \pm 91.6$ & $277.1 \pm 67.5$ & $493.6 \pm 106.7$ & $85.4 \pm 11.7$ \\
\hline \multicolumn{5}{|l|}{ Age } \\
\hline 6 to 10 years & $446.1 \pm 72.9$ & $215.4 \pm 52.1$ & $428.7 \pm 96.0$ & $89.6 \pm 10.4$ \\
\hline 11 to 14 years & $526.8 \pm 63.5^{* *}$ & $275.0 \pm 49.3^{* *}$ & $503.2 \pm 87.5^{* *}$ & $84.7 \pm 10.5^{* *}$ \\
\hline 15 to 19 years & $582.1 \pm 81.4^{* *}$ & $326.3 \pm 47.5^{* *}$ & $538.4 \pm 106.1^{* *}$ & $82.6 \pm 13.6^{* *}$ \\
\hline \multicolumn{5}{|l|}{$B M l$} \\
\hline $\begin{array}{l}\text { Not overweight or } \\
\text { obese }\end{array}$ & $523.5 \pm 91.3$ & $277.1 \pm 68.3$ & $495.4 \pm 107.2$ & $85.2 \pm 11.5$ \\
\hline $\begin{array}{l}\text { Overweight or } \\
\text { obese }\end{array}$ & $524.6 \pm 93.1$ & $277.0 \pm 64.2$ & $487.1 \pm 105.0$ & $86.2 \pm 12.5$ \\
\hline
\end{tabular}

included a wider range of sedentary time variables than what has been previously considered that characterize the timing and patterning of how the sedentary time is accumulated. Further, the sedentary pattern variables were designed to be more reflective of real-world sedentary behaviour. For example, a limited number of short transitions into light activity were allowed to reflect real-life situations where individuals are sedentary for long periods but move around occasionally (i.e., to go to the washroom or answer the phone). Despite the inclusion of more comprehensive sedentary pattern variables, this study found few significant relationships with health risk and the associations observed were limited to boys aged 11-14 years.

In theory, excessive sedentary time is associated with negative health outcomes [4,37] and self-reported screen time is associated with elevated health risk in children [1-3]; however, the way we currently measure sedentary time with accelerometers does not consistently support this link. To date, the research linking accelerometermeasured sedentary time with health risk among children and youth has been mixed. It is therefore unclear whether a relationship exists only in some populations or if differences in analytical approaches explain the inconsistencies observed. There appears to be more evidence supporting a lack of relationship between accelerometer-measured sedentary time and health risk in children and youth [1,11-16] than there is supporting a relationship [8-10]. Adjustment for MVPA appears to attenuate significant associations between accelerometer-measured sedentary time and health risk [13,16], suggesting that MVPA is more powerful than total sedentary time at explaining the variance in health risk in children and youth. In our unadjusted regression models, sedentary time was associated with BMI and waist circumference in boys aged 6 to 14 and girls aged 6 to 10 years; however, after adjustment for MVPA, these associations remained significant only in 11-14 year old boys.

In 2008, Healy and colleagues published a paper that reported a significant association between number of daily breaks in accelerometer-measured sedentary time and lower metabolic risk in adults [17]. This work led researchers to question whether it is the pattern of how sedentary time is accumulated, rather than simply the total volume of sedentary time, which matters for health. Do frequent interruptions in sedentary time attenuate the health risk that sedentary time imposes? Does this relationship apply in both children and adults? Carson and Janssen found no significant associations between breaks in sedentary time or prolonged bouts of sedentary time lasting 30 minutes with cardio-metabolic risk 
factors in a large sample of American children [1]. Number of breaks in sedentary time was only associated with waist circumference in 11-14 year old boys in the present analysis. We included an additional layer of complexity by examining sedentary time, breaks and prolonged bouts of sedentary time during periods of discretionary free time: weekends and after school. We hypothesized that sedentary time accumulated during these periods would better discriminate between children engaging in healthy and unhealthy levels of sedentary behaviour when compared to simply examining overall sedentary time. We observed no significant associations between the patterns of sedentary time accumulated on weekends and health risk in children; however, some relationships emerged when we examined sedentary time accumulated during the after school period. Interestingly, we only observed significant associations in boys aged 11 to 14 years of age when the regression models were adjusted for age, MVPA and accelerometer wear time.

It is difficult to speculate why we observed significant findings in boys and not girls. It is possible that more overweight and obese boys in this sample were engaging in prolonged bouts of sedentary time after school, a finding consistent with previous research that has found that boys spend considerably more time in specific sedentary behaviours such as video game playing [38-40]. Average daily sedentary time and weekend sedentary time did not differ between boys and girls while sedentary time accumulated after $3 \mathrm{pm}$ on weekdays was higher in boys compared to girls (277 vs. 266 minutes). Significant differences between boys who are not overweight/obese and overweight/obese boys were observed in the sedentary variables; however, no such differences were observed in girls. For example, there was virtually no difference in average daily sedentary time between girls who are not overweight/obese versus those who are (524 vs. $525 \mathrm{~min} \cdot \mathrm{d}^{-1}$ ) while a more marked difference existed between boys who are not overweight/obese versus those who are (500 vs. $\left.528 \mathrm{~min} \cdot \mathrm{d}^{-1}\right)$. In Figure 1, a distinction between boys who are not overweight/obese and those who are can be observed across all bout lengths; however the difference is only statistically significant when the bout length is at least 80 minutes long. By comparison, no difference is noticeable by overweight/obesity status in girls and there is more crossover in the error bars in girls (Figure 2). Similarly, no significant associations emerged in girls in the regression analyses while in 11-14 year old boys, prolonged bouts of sedentary time lasting at least 80 minutes, accumulated during the after school period were associated with both BMI and waist circumference. Explaining why significant associations were observed in 11-14 year olds boys but not those who were 6-10 or 15-19 years is not easy. In a large sample of US children, Sisson and colleagues observed an increase in screen time with age from 2 to 15 years [41]. In the Health Behaviour and School Aged Children Survey, the Canadian data show that screen time increases from age 11 to 15 years [42] with the peak occurring in grade 9 (approximately 14 years) [43]. These large data sets suggest that the 1114 year old age group may be an age range where screen time habits change significantly, thus increasing the amount of variation (and likelihood to find significant associations) in this variable.

The lack of evidence linking accelerometer-measured sedentary time with health risk in children is counterintuitive given the consistent observation that screen time, a key contributor to total sedentary time, is associated with health risk [1-4]. One of the fundamental differences between self-reported screen time and objectively measured sedentary time is that the former is capturing one specific activity while the latter is capturing screen time in addition to many other sedentary behaviours. Much of the time accumulated as "sedentary" represents normal aspects of day-to-day life therefore capturing every minute in a day that is sedentary, as accelerometers do, may dilute the associations between specific sedentary behaviours (e.g., watching television) and health risk. It is possible that some forms of sedentary behavior (e.g., screen time, long car or bus travel) are associated with negative health outcomes while other forms of sedentary behavior (e.g., eating, reading, resting, socializing etc.) are not. Similarly, data reduction procedures used in accelerometry analysis (e.g., 10 hour wear time criteria) were developed to accurately capture MVPA and whether they are appropriate for sedentary behaviour research questions is unknown. For example, it has been suggested that wear time has a disproportionate impact upon estimates of sedentary time compared with MVPA [44]. Teasing out which sedentary behaviours beyond screen time are associated with negative health outcomes represents an important area for future research.

The sedentary behaviours that are of known public health concern in children and youth (e.g., excessive levels of screen time) typically last for extended periods of time (i.e., up to several hours at once). This reality was the motivation behind the way prolonged bouts of sedentary time were defined in the present analysis. Had we used a strict definition of what ended about (i.e., any transition out of sedentary) then our longest bout length would have been very short (e.g., 10 minutes) and thus not representative of one of the key sedentary behaviours that we were interested in capturing. The allowance of a modest amount of light intensity movement within the prolonged sedentary bouts was therefore purposeful and allowed much longer bout lengths to be 
examined (up to 2 hours). Number of breaks per day, also assessed in this analysis, is an important aspect of sedentary behaviour patterns. Given that we and others [1] have not consistently observed significant associations between number of breaks per day and health risk, it is important to look at alternative pattern variables such as prolonged bouts. Further, the extension of bout length in the present analysis was important to build off the only other published work that examined prolonged bouts up to 30 minutes in children and youth [1].

It is possible that the true health effect of sedentary time is attenuated by limitations with the data and analysis. Possible limitations that dilute the possibility of observing a true relationship include: i) the cross-sectional nature of the data, ii) non-response bias, iii) the possibility that the findings in 11-14 year olds boys reflect Type 1 error. As described in the methodology, the nonresponse bias is adjusted for in the data. We attempted to minimize the likelihood of Type 1 errors in the regression analyses by adjusting the $\mathrm{p}$-value for significance from .05 to .006 . Other limitations include the lack of ability to confirm precisely when children were finished school. We examined the period after $3 \mathrm{pm}$ on weekdays [45] based on the assumption that most kids would finish school sometime between 2-4 pm. Accelerometers are limited in their ability to capture postural changes (i.e., cannot differentiate between sitting and standing) and are therefore limited in their ability to measure sedentary before as well as other tools which encompass an inclinometer in addition to an accelerometer. No significant associations were observed between sedentary time variables and blood pressure or nonHDL cholesterol and this may be due to it likely being more difficult to detect meaningful differences in biomarkers in children and youth than adults because younger people are more distal to pathophysiological developments. A similar examination on a population of high-risk children (e.g., overweight or obese or with a family history of cardio-metabolic disease) may lead to different findings as these children would be more likely to exhibit abnormalities in blood markers and blood pressure. Finally, examination of interaction and confounding effects was limited because the number of variables (including interaction terms) that can be tested within the CHMS data set is limited by the available degrees of freedom.

\section{Conclusions}

Sedentary time accumulated during the after school period was associated with BMI and waist circumference, independent of MVPA, in boys aged 11 to 14 years. No sedentary behaviour variables were independently associated with any health markers in older or younger boys or in girls of any age. Future studies should consider examining more comprehensive sedentary time pattern variables when attempting to elucidate the relationships between sedentary time and health risk in children and youth.

\section{Additional files}

\begin{abstract}
Additional file 1: Table S1. Associations between sedentary time variables and body mass index, presented by sex and age groups.

Additional file 2: Table S2. Associations between sedentary time variables and waist circumference, presented by sex and age groups. Additional file 3: Table S3. Associations between sedentary time variables and systolic blood pressure, presented by sex and age groups.

Additional file 4: Table S4. Associations between sedentary time variables and diastolic blood pressure, presented by sex and age groups.

Additional file 5: Table S5. Associations between sedentary time variables and non-HDL cholesterol, presented by sex and age groups.
\end{abstract}

\section{Competing interests}

The authors declare that they have no competing interests.

\section{Authors' contributions}

RCC conceived the manuscript, formed the research team, directed the analysis, led the writing. DG was involved in the conception the manuscript, completed the analysis and contributed to the writing. IJ contributed to the writing and provided critical review of the analysis. SLW was involved in the conception of the manuscript, contributed to the writing and provided critical review of the analysis and writing. TJS, VC and MST contributed to the writing and provided critical review of the writing. All authors read and approved the final manuscript.

\section{Acknowledgements}

The authors would like to thank the participants of the Canadian Health Measures Survey as well as all staff at Statistics Canada involved in the operations of the survey.

\section{Author details}

${ }^{1}$ Health Analysis Division, Statistics Canada, Ottawa, ON, Canada. ${ }^{2}$ Children's Hospital of Eastern Ontario Research Institute, Ottawa, ON, Canada. ${ }^{3}$ School of Kinesiology and Health Studies, Queen's University, Kingston, ON, Canada. ${ }^{4}$ Department of Community Health and Epidemiology, Queen's University, Kingston, ON, Canada.

Received: 25 October 2012 Accepted: 22 February 2013 Published: 7 March 2013

\section{References}

1. Carson V, Janssen I: Volume, patterns, and types of sedentary behavior and cardio-metabolic health in children and adolescents: a crosssectional study. BMC Public Health 2011, 11:274.

2. Mark $A E$, Janssen I: Relationship between screen time and metabolic syndrome in adolescents. J Public Health (Oxf) 2008, 30:153-160.

3. Sisson SB, Church TS, Martin CK, Tudor-Locke C, Smith SR, Bouchard C, Earnest CP, Rankinen T, Newton RL, Katzmarzyk PT: Profiles of sedentary behavior in children and adolescents: The US National Health and Nutrition Examination Survey, 2001-2006. Int J Pediatr Obes 2009, 4(4): 353-359.

4. Tremblay MS, Leblanc AG, Kho ME, Saunders TJ, Larouche R, Colley RC, Goldfield G, Connor Gorber S: Systematic review of sedentary behaviour and health indicators in school-aged children and youth. Int J Behav Nutr Phys Act 2011, 8:98.

5. Sedentary Behaviour Research Network: Letter to the editor: standardized use of the terms "sedentary" and "sedentary behaviours". Appl Physiol Nutr Metab 2012, 37(3):540-542.

6. Adamo KB, Prince SA, Tricco AC, Connor-Gorber S, Tremblay MS: A comparison of indirect versus direct measures for assessing physical 
activity in the pediatric population: a systematic review. Int J Pediatr Obes 2009, 4(1):2-27.

7. Colley RC, Wong SL, Garriguet D, Janssen I, Connor Gorber S, Tremblay MS Physical activity, sedentary behaviour and sleep in Canadian children: A comparison between parent-report and accelerometry measures and their relative association with health outcomes. Health Rep 2012, 23(2):1-9.

8. Celis-Morales CA, Perez-Bravo F, Ibanez L, Salas C, Bailey MES, Gill JMR: Objective vs. Self-reported physical activity and sedentary time: Effects of measurement method on relationships with risk biomarkers. PLoS One 2012, 7(5):e36345.

9. Martinez-Gomez D, Eisenmann JC, Gomez-Martinez S, Veses A, Marcos A, Veiga OL: Sedentary behavior, adiposity, and cardiovascular risk factors in adolescents. The AFINOS Study. Rev Esp Cardiol 2010, 63:277-285.

10. Sardinha LB, Andersen LB, Anderssen $S A$, Quiterio AL, Ornelas R, Froberg $K$ Anderssen SA, Riddoch CJ, Quiterio AL, Ekelund U: Objectively measured time spent sedentary is associated with insulin resistance independent of overall and central body fat in 9- to 10-year-old Portuguese children. Diabetes Care 2008, 31(3):569-575

11. Chaput JP, Lambert M, Mathieu ME, Tremblay MS, O'Loughlin J, Tremblay A: Physical activity vs. sedentary time: independent associations with adiposity in children. Pediatr Obes 2012, 7(3):251-258.

12. Chinapaw MJM, Yildirim M, Altenburg TM, Singh AS, Kovacs E, Molnar D, Brug J: Objective and self-rated sedentary time and indicators of metabolic health in Dutch and Hungarian 10-12 year olds: The ENERGY project. PLoS One 2012, 7(5):e36657.

13. Ekelund U, Luan J, Sherar LB, Esliger DW, Griew P, Cooper A, for the International Children's Accelerometry Database (ICAD) Collaborators: Moderate to vigorous physical activity and sedentary time and cardiometabolic risk factors in children and adolescents. JAMA 2012, 307(7):704-712.

14. Martinez-Gomez D, Eisenmann JC, Healy GN, Gomez-Martinez S, Diaz LE, Dunstan DW, Veiga OL, Marcos A, AFINOS Study Group: Sedentary behaviors and emerging cardiometabolic biomarkers in adolescents. J Pediatr 2012, 160(1):104-110.

15. Martinez-Gomez D, Tucker J, Neelen KA, Welk GJ, Eisenmann JC: Associations between sedentary behavior and blood pressure in young children. Arch Pediatr Adolesc Med 2009, 163:724-730.

16. Steele RM, van Sluijs EMF, Cassidy A, Griffin SJ, Ekelund U: Targeting sedentary time or moderate- and vigorous-intensity activity: independent relations with adiposity in a population-based sample of 10-y-old British children. Am J Clin Nutr 2009, 90:1185-1192.

17. Healy GN, Dunstan DW, Salmon J, Cerin E, Shaw JE, Zimmet P, Owen N: Breaks in sedentary time - Beneficial associations with metabolic risk. Diabetes Care 2008, 31(4):661-666.

18. Atkin AJ, Gorely T, Biddle SJ, Marshall SJ, Cameron N: Critical hours: physical activity and sedentary behaviour of adolescents after school. Pediatr Exerc Sci 2008, 20:446-456.

19. Fairclough SJ, Burcher ZH, Stratton G: Whole-day and segmented-day physical activity variability of northwest England school children. Prev Med 2007, 44:421-425.

20. Harrington DM, Dowd KP, Bourke AK, Donnelly AE: Cross-sectional analysis of levels and patterns of objectively measured sedentary time in adolescent females. Int J Behav Nutr Phys Act 2011, 8:120.

21. Day B, Langlois R, Tremblay MS, Knoppers B-M: Canadian Health Measures Survey: Ethical, legal and social issues. Health Rep 2007, 18:37-51.

22. Tremblay MS, Wolfson M, Connor Gorber S: Canadian Health Measures Survey: background, rationale and overview. Health Rep 2007, 18(Suppl):7-20.

23. Colley RC, Garriguet D, Janssen I, Craig CL, Clarke J, Tremblay MS: Physical activity of Canadian children and youth: Accelerometer results from the 2007 to 2009 Canadian Health Measures Survey. Health Rep 2011, 22(1):15-23.

24. Colley RC, Gorber SC, Tremblay MS: Quality control and data reduction procedures for accelerometry-derived measures of physical activity. Health Rep 2010, 21(1):63-69.

25. Puyau MR, Adolph AL, Vohra FA, Zakeri I, Butte NF: Prediction of activity energy expenditure using accelerometers in children. Med Sci Sports Exerc 2004, 36(9):1625-1631.

26. Wong SL, Colley RC, Connor Gorber S, Tremblay MS: Actical accelerometer sedentary activity threshold for adults. J Phys Act Health 2011, 8:587-591.
27. Cole TJ, Bellizzi MC, Flegal KM, Dietz WH: Establishing a standard definition for children overweight and obesity worldwide: international survey. BMJ 2000, 320:1240.

28. Canadian Society for Exercise Physiology: The Canadian Physical Activity, Fitness and Lifestyle Approach (CPAFLA). 3rd edition. Ottawa, ON: Canadian Society for Exercise Physiology; 2004.

29. Bryan S, Saint-Pierre Larose M, Campbell N, Clarke J, Tremblay MS: Resting blood pressure and heart rate measurement in the Canadian Health Measures Survey, cycle 1. Health Rep 2010, 21(1):71-78.

30. Myers MG, Valdivieso MA: Use of an automated blood pressure recording device, the BpTRU, to reduce the "while coat effect" in routine practice. Am J Hypertens 2003, 16(6):494-497

31. Gardner CD, Winkleby MA, Fortmann SP: Population frequency of nonhigh-density lipoprotein cholesterol (Third National Health and Nutrition Examination Survey [NHANES III], 1988-1994). Am J Cardiol 2000, 86(3):299-304

32. Liu J, Joshi D, Sempos CT: Non-high-density lipoprotein cholesterol and cardiovascular risk factors among adolescents with and without impaired fasting glucose. Appl Physiol Nutr Metab 2009, 34(2):136-142.

33. Katmarzyk PT: Waist circumference percentiles for Canadian youth 11-18y of age. Eur J Clin Nutr 2004, 58(7):1011-1015.

34. Rao JNK, Wu CFJ, Yue K: Some recent work on resampling methods for complex surveys. Surv Meth 1992, 18(2):209-217. Statistics Canada, Catalogue 12-001.

35. Rust KF, Rao JNK: Variance estimation for complex surveys using replication techniques. Stat Methods Med Res 1996, 5:281-310.

36. Statistics Canada. Canadian Health Measures Survey (CHMS) Data User Guide: Cycle 1. 2010. Available at: http://www23.statcan.gc.ca/imdb-bmdi/ pub/document/5071_D2_T1_V1-eng.htm. Accessed March 11, 2013.

37. Tremblay MS, Colley RC, Saunders TJ, Healy GN, Owen N: Physiological and health implications of a sedentary lifestyle. Appl Physiol Nutr Metab 2010, 35:725-740

38. Goldfield GS, Kenny GP, Hadjiyannakis S, Phillips P, Alberga AS, Saunders TS, Tremblay MS, Malcolm J, Prud'homme D, Gougeon R, Sigal RJ: Video game playing independently associated with blood pressure and lipids in overweight and obese adolescents. PLoS One 2011, 6(11):e26643.

39. Granich J, Rosenberg M, Knuiman MW, Timperio A: Individual, social, and physical environment factors associated with electronic media use among children: sedentary behavior at home. J Phys Act Health 2011, 8(5):613-625.

40. Marshall SJ, Gorely T, Biddle SJ: A descriptive epidemiology of screenbased media use in youth: a review and critique. J Adolesc 2006, 29(3):333-349.

41. Sisson SB, Church TS, Martin CK, Tudor-Locke C, Smith SR, Bouchard C, et al: Profiles of sedentary behavior in children and adolescents: the US National Health and Nutrition Examination Survey, 2001-2006. Int Pediatr Obes 2009, 4(4):353-359.

42. Currie C, Zanotti C, Morgan A, Currie D, de Looze M, Roberts C, et al (Eds): Social determinants of health and well-being among young people. Health Behaviour in School-aged Children (HBSC) study: international report from the 2009/2010 survey, Health Policy for Children and Adolescents, No. 6. Copenhagen: WHO Regional Office for Europe; 2012.

43. Active Healthy Kids Canada: Is Active Play Extinct - The 2012 Active Healthy Kids Canada Report Card on Physical Activity for Children and Youth. Toronto: Active Healthy Kids Canada; 2012

44. Tudor-Locke C, Jonson WD, Katzmarzyk PT: U.S. Population profile of timestamped accelerometer outputs: Impact of wear time. J Phys Act Health 2011, 8:693-698.

45. Fairclough SJ, Beighle A, Erwin H, Ridgers ND: School day segmented physical activity patterns of high and low active children. BMC Public Health 2012, 12:406.

\section{doi:10.1186/1471-2458-13-200}

Cite this article as: Colley et al.: The association between accelerometermeasured patterns of sedentary time and health risk in children and youth: results from the Canadian Health Measures Survey. BMC Public Health 2013 13:200 